\title{
A REVIEW REPORT ON NANOMEDICINE AND DIABETES
}

\author{
Nandakumar Ravichandran \\ Department of Biomedical Engineering \\ PSG College of Technology, Coimbatore, Tamilnadu, Chennai
}

\begin{abstract}
Nanomedicine is a branch of science that combines medicine and nanotechnology for diagnosing, sensing, delivery, and treatment purposes. It improves the ability to target specific cells and tissues. Their features such as biocompatibility, increased retention and reactivity, and low cytotoxicity make them find their application in the field of Diabetic therapy. A methodology on treating diabetes using medical nanotechnology is proposed in this paper and the methodology is yet to be tested.
\end{abstract}

Keywords - Diabetes, stem cell, nanocapsule, nanosensor, nanogripper, beta cell, nanomedicine

\section{INTRODUCTION}

Diabetes mellitus (Diabetes) is the seventh leading cause of death globally. Between 2000 and 2016, premature mortality from diabetes has increased by five percent. In 2019, an estimated 1.5 million deaths were caused by diabetes. And today, in this 21st century, Nanotechnology based Stem Cell Therapy which is an application of Nanomedicine acts as a promising cure for diabetes. This is due to the inert, biocompatibility nature of the selected nanomaterial and potential of the stem cell to develop into a tissue/organ. In this paper, we will discuss Nanotechnology based Stem Cell Therapy, recent advancements, researches, and future works in this field.

\section{NOMENCLATURE}

\section{A. Nanomedicine -}

Nanomedicine refers to the applications of nanomaterials in medicine. It is preferred due to its grain size. Small amount of drug volume is enough to treat and there is an increase in the drug target specificity. They stay in the body for a long time and provide sufficient response. Imaging applications involve MRI, ultrasound, etc. Sensing applications involve biosensors to detect cancers such as breast cancer and also used to detect the genetic sequence of living beings. Delivery applications in treatment involve abraxane for cancer patients, cabenuva for HIV-1 patients, etc. Suping $\mathrm{Li}$ et al. ${ }^{1}$, constructed an autonomous DNA robot programmed to transport payloads and present them specifically in tumors, where the intravenously injected DNA nanorobots induce thrombin at the tumor site, inhibiting tumor growth and resulting in tumor necrosis.

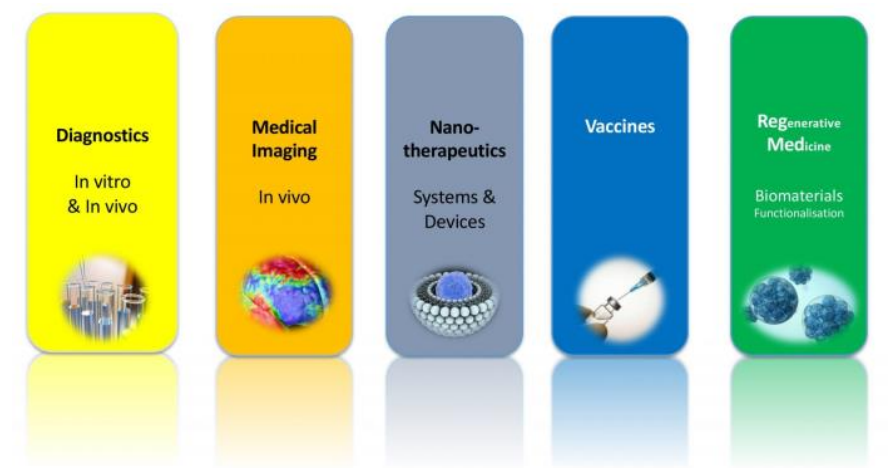

Fig. 1. Applications of Nanomedicine

\section{B. Stem cell -}

Stem cell is the type of cell which can develop into specialized cell types, ranging from muscle cells to brain cells. There are different types of stem cells - embryonic stem cells, cord stem cells and adult stem cells. Embryonic stem cells and Cord stem cells are pluripotent - can develop into any specialized types of cells. Adult stem cells are multipotent - can develop into specific types of cells. The next generation of stem cells is human induced pluripotent stem cells which are derived from adult human skin. Some of the advances in research include Haemopoietic Stem Cell Transplantation (E.B. Austin et al. ${ }^{2}$ ), Assisted Reproductive Technologies such as Mitochondrial Replacement Technique (Yeonmi Lee et al. $^{3}$ ), wound healing (J. Michael Sorrell et al. ${ }^{4}$ ), etc. 


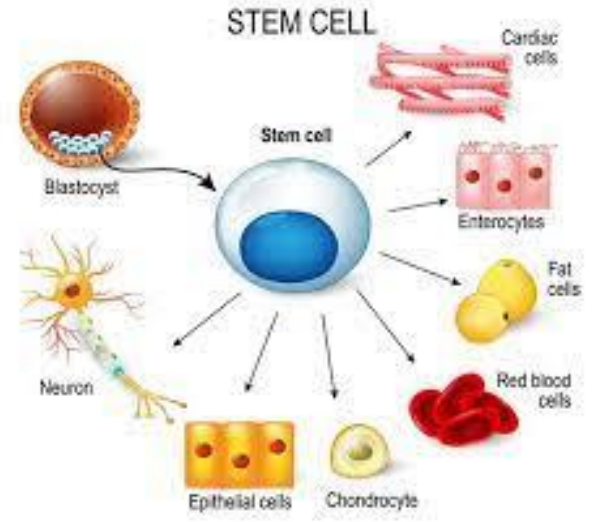

Fig. 2. Stem cell and specialised cells

\section{Diabetes -}

Diabetes is a metabolic disorder where the patients suffer from insufficient insulin production, or the pancreas cannot use the insulin effectively. It is a chronic condition which leads to a series of diseases and ailments such as cardiovascular diseases, Diabetic Retinopathy, impaired glucose tolerance, etc. The different types of diabetes are namely T1 Dependent, T2 Dependent, gestational diabetes and prediabetes.

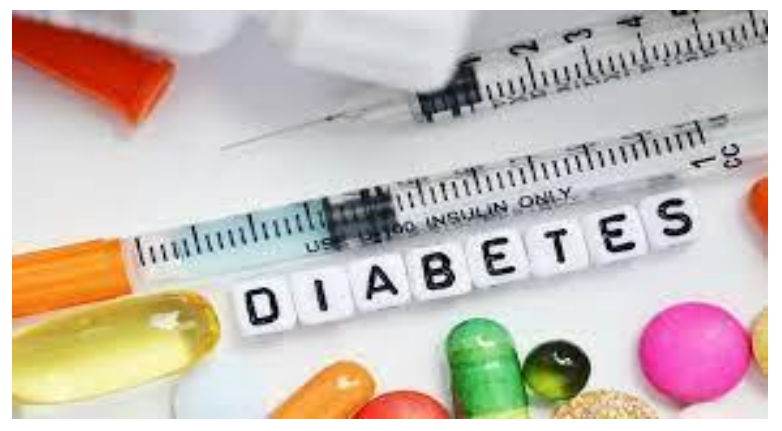

Fig. 3. Diabetes and treatment

\section{NANOTECHNOLOGY BASED STEM CELl THERAPY For} DIABETES

\section{A. Apparatus -}

The components are

- Encapsulated nanomaterial

- Nanosensor

- Nanogrippers

- Polymeric nanocapsule

The components are designed using 3D modelling software.

\section{1) Encapsulated nanomaterial}

Nanomaterial of suitable size and shape is designed. Polyhedral gold and polymer nanomaterials are preferred. According to Yu-Ning $\mathrm{Wu}$ et al. ${ }^{5}$, Polyhedral nanoparticles i.e., Dodecahedral and octa decahedron nanoparticles exhibit better structural, thermal and shape stability. The nanomaterial is semi-permeable to the transport of $\mathrm{O} 2$ and other nutrients. The nanomaterial will be encapsulated with suitable material such as alkyl thiols, alkane thiolates, arene thiolate, DNA, protein, or enzymes. The works of Rick de Vries et al. ${ }^{6}$, describe the advantages of nanoencapsulation such as improvement in mass transport of essential nutrients to the islets and in reducing the total volume of material and cells that are transplanted.

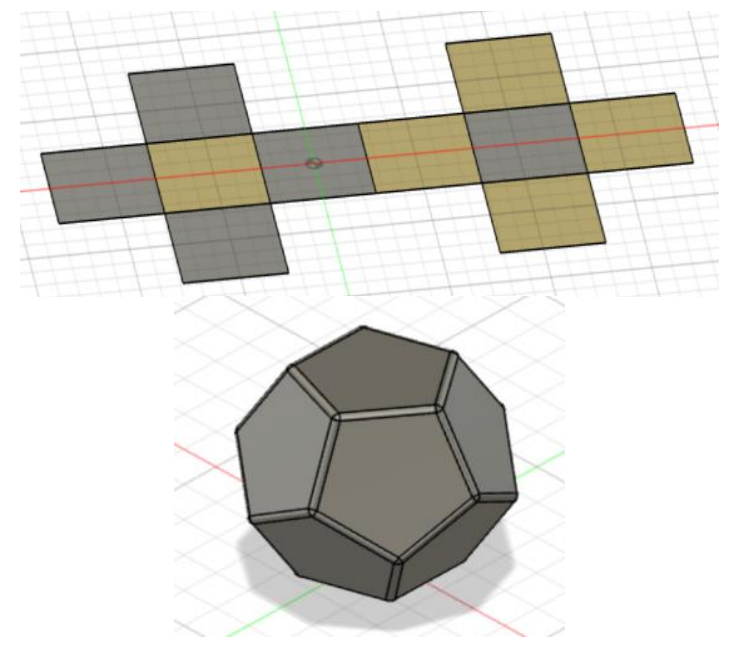

Fig. 4. Nanomaterial - Polyhedron structure (Dodecahedron)

\section{2) Nanosensor}

Nanosensor chips are built by combining sensors on silicon chips for monitoring factors such as temperature, pressure, $\mathrm{pH}$, etc. The same is designed to detect cancer biomarkers, etc. Lingyun $\mathrm{Xu}$ et al. ${ }^{7}$, fabricated an array of magneto nanosensors that employ the effect of giant magnetoresistance to perform multiplexed assays with a single chip and demonstrated to detect radiation-exposure biomarkers, autoantibodies, and cancer biomarkers. According to Anam Munawar et al. ${ }^{8}$, the deployment of highly sensitive and selective nanosensors can detect disease, toxins, or biological threats (tumors, cancers, etc.) and create significant improvements in clinical as well as environmental outcomes. 


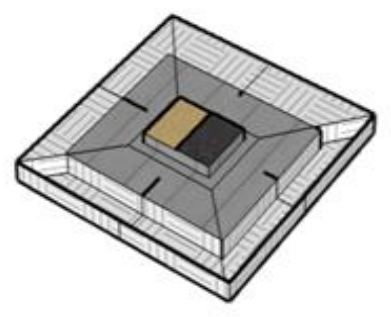

Fig. 5. Nanosensor

\section{3) Nanogrippers}

Nanogrippers are nanostructures which can lift a cell or an object from a surface freely. It can also be used to hold an object in a position. The team led by John Hopkin University scientists used microgrippers to remove tiny samples from bile ducts of pig. Pedram Heidari et al. ${ }^{9}$ in their paper discussed the high capability of displacing objects of different sizes and weights by shape memory alloys grippers. The nanogrippers implemented in the system have the potential to detect tumors such as teratomas by capturing it and sending it to a gold standard test i.e., biopsy.

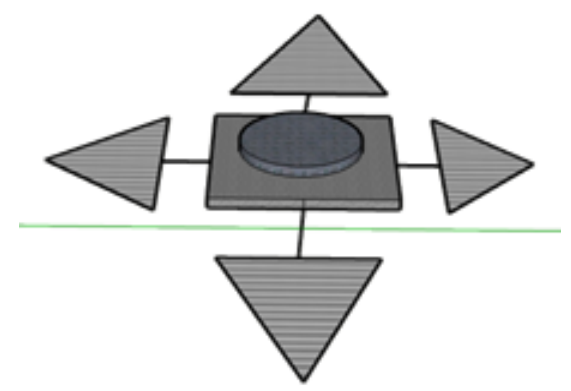

Fig. 6. Nanogrippers

\section{4) Polymeric Nanocapsule}

According to Radhika et al. ${ }^{11}$, polymeric nanocapsule can be made in specific shapes, sizes and in reasonable quantities. They can be used as smart drugs because they can be made to bind to specific cells. The advantages are sustained release, incremental drug selectivity and effectiveness, improvement of drug bioavailability and alleviation of drug toxicity. This makes the nanocapsule find application in targeting the teratomas if detected by the sensor and biopsy.

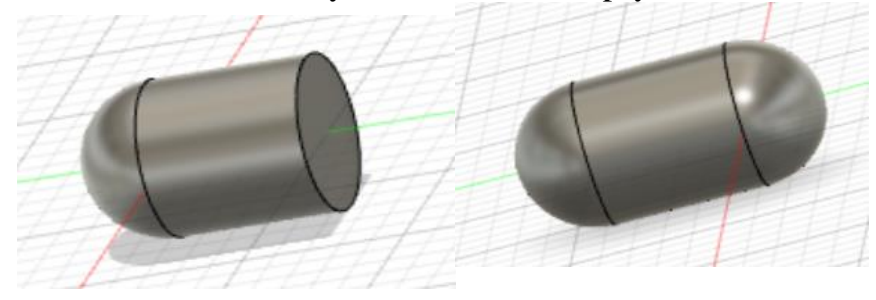

Fig. 7. Polymeric Nanocapsules

\section{B. Proposed Methodology -}

Skin cells obtained from the subject can be reprogrammed into induced Pluripotent Stem Cells (iPSCs) by activating the cells' own genes (Jere Weltner et al. ${ }^{13}$ ). These iPSCs have the potential to develop into specialized cell types. Undifferentiated beta cells are obtained by adding growth factors to the iPSCs and giving a stimulus if needed (Yasushi et al. ${ }^{14}$, Pagliuca et al. ${ }^{15}$, Jefferey et al. ${ }^{16}$ ). The undifferentiated beta cells are packed along with the nanosensor, nanogrippers and nanocapsule inside an encapsulated nanomaterial. The nanomaterial layer is designed in a way such that it is semipermeable to $\mathrm{O}_{2}$ and other nutrients ( $<1$ micron) and impermeable to immune cells ( $>7$ microns). These undifferentiated beta cells begin to differentiate and once they got differentiated, they are released. The differentiated cells start producing insulin and begins to function effectively. Sometimes there can be occurrence of teratomas, a rare type of tumor because of the fully developed beta cells. Nanosensors deployed, will constantly monitor the temperature, $\mathrm{pH}$, and other conditions. Once the $\mathrm{pH}$ is found to be slightly lower or any unfavorable conditions detected like cancer biomarkers, the nanogrippers deployed will capture a cell inside and send for biopsy to confirm the presence/ prevalence of teratomas, if needed. It is signaled to induce apoptosis by releasing drugs present inside the nanocapsule in a controlled manner if teratomas are diagnosed.

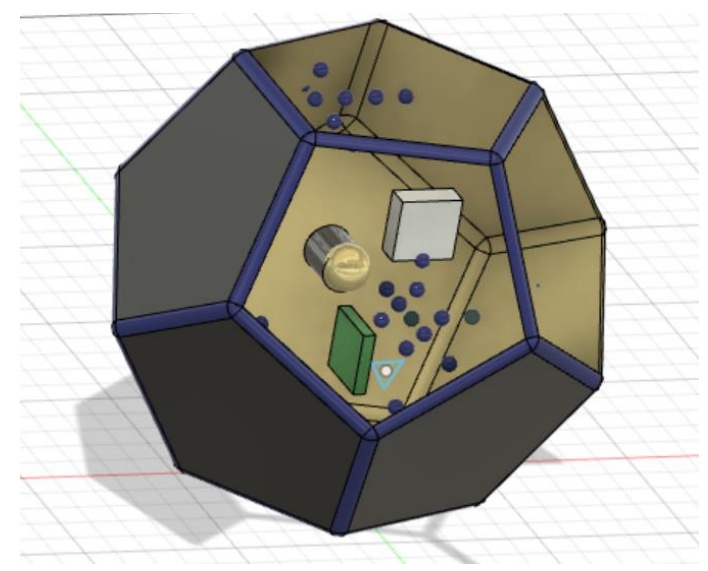

Fig. 8. Nanomaterial packed up with undifferentiated beta cells, nanosensor, nanogrippers and nanocapsule 


\section{International Journal of Engineering Applied Sciences and Technology, 2021 Vol. 6, Issue 1, ISSN No. 2455-2143, Pages 190-194 \\ Published Online May 2021 in IJEAST (http://www.ijeast.com)}

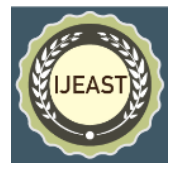

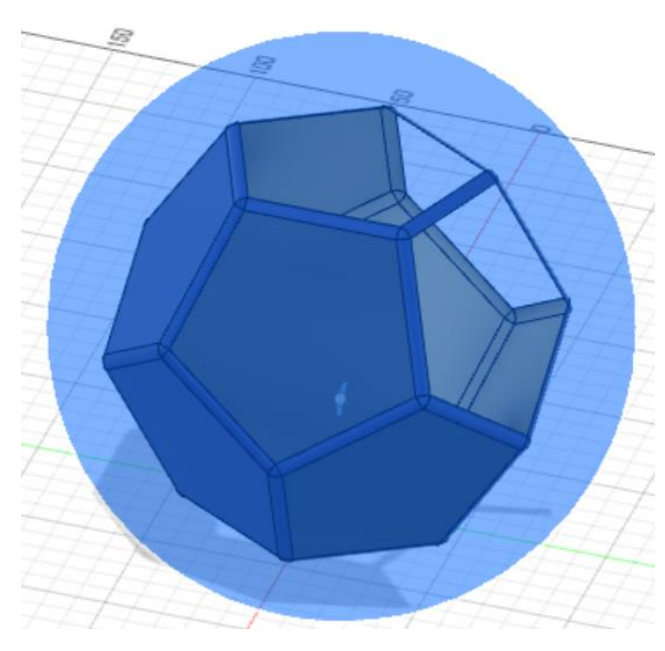

Fig. 9. Encapsulated nanomaterial

\section{ADVANTAGES AND HINDRANCES}

The proposed methodology ensures biocompatibility, protecting stem cells from destruction. The encapsulation of the nanomaterial protects the stem cell from agglomeration. This also rejects the need for immunosuppressive drugs since the stem cell is derived from the same subject. The deployment of nanosensor and nanogrippers is useful in detecting the occurrence of teratomas. Nanocapsule induces apoptosis and prevent the growth and occurrence of teratomas by releasing the drugs loaded. Some of the hurdles are longevity and the glucose response should match that of normal adult beta cells.

\section{FUTURE WORKS}

Nanomaterials to be designed using methods such as chemical vapor deposition, photolithography, etc. Pluripotent stem cells must be generated and programmed to obtain beta cells. Clinical trials of proposed methodology are to be performed.

\section{CONCLUSION}

Application of nanomedicine in treating diabetes has been one of the trending topics in today's century. Future works on this area of research can be persuaded. The same proposed methodology can be applied in treating various diseases like neurological diseases, cancer, etc. Other applications of nanomedicine include nanomaterial based targeted drug therapy, tissue engineering, antibiotic resistance, etc.

\section{REFERENCES}

[1] Suping Li, Qiao Jiang, Shaoli Liu, Yinlong Zhang, Yanhua Tian, Chen Song, Jing Wang, Yiguo Zou, Gregory J Anderson, Jing-Yan Han, Yung Chang, Yan Liu, Chen Zhang, Liang Chen, Guangbiao Zhou, Guangjun Nie, Hao Yan, Baoquan Ding \& Yuliang Zhao, "A DNA nanorobot functions as a cancer therapeutic in response to a molecular trigger in vivo", Nature Biotechnology, 36, 258-264, February 2018.

[2] E.B. Austin, M.Guttridge, D.Pamphilon and S.M. Watt, "The role of blood services and regulatory bodies in stem cell transplantation", Vox Sanguinis, 94(1), 6-17, January 2008.

[3] Yeonmi Lee and Eunju Kang, "Stem cells and reproduction", BMB Reports, 52(8), 482-489, August 2019.

[4] J. Michae Sorrell, Marilyn A. Baber, and Arnold I. Caplan, "Influence of Adult Mesenchymal Stem Cells on Invitro Vascular Formation", Tissue Engineering, 15(7), January 2009.

[5] Yu-NingWu, RaoHuang, Xiang-MingZeng and YuHuaWen, "Octadecahedral and dodecahedral iron nanoparticles: An atomistic simulation on stability and shape evolutions," Physics Letters A,380(5), October 2015.

[6] Rick de Vries, Adam Stell, Sami Gemal Mohammed, Carolin Hermanns, Adela Helvia Martinez Sanchez, Marlon J.A. Jetten and Aart Van Apeldoorn, "Bioengineering, biomaterials, and $\beta$-cell replacement therapy," Transplantation, Bioengineering, and Regeneration of the Endocrine Pancreas, Vol.2,461486,January 2020.

[7] Lingyun Xu, Jung-Rok Lee, Shiying Hao, Xuefeng Bruce Ling, James D. Brooks, Shan X. Wang, and Sanjiv Sam Gambhir, "Improved detection of prostate cancer using a magneto-nanosensor assay for serum circulating autoantibodies,"PLos One, 14(8), August 2019.

[8] Anam Munawar, Yori Ong, Romana Schirhagl, Muhammad Ali Tahir, Waheed S. Khanae and Sadia Z. Bajwa,"Nanosensors for diagnosis with optical, electric and mechanical transducers," $R S C$ Advances, 9, Issue 12,February 2019.

[9] Pedram Heidari , Majid Salehi and Mojtaba Kolahdoozan,"An overview of Micro Grippers used in Micro-Nano-Mechanical Systems and comparing the Results in Medical Applications",ICME,October 2018.

[10] “Microscopic Star-Shaped Grippers Take Biopsies,” NIH Research Matters, May 2013.

[11] R.Radhika, Sasikanth and T.Sivakumar , "Nanocapsules: A new approach in drug delivery," International Journal of Pharmaceutical sciences and research ,1426-1429, June 2011. 
[12] Nandakumar Ravichandran, "Stem Cell Therapy for T1D and T2D Diabetic Patients," International Journal of Scientific \& Engineering Research, 11(10),23-25,October 2020.

[13] Jere Weltner, Diego Balboa, Shintaro Katayama, Maxim Bespalov, Kaarel Krjutškov, Eeva-Mari Jouhilahti, Ras Trokovic, Juha Kere \& Timo Otonkoski, "Human pluripotent reprogramming with CRISPR activators," Nature Communications, 9, July 2018.

[14] Yasushi Kondo, Taro Toyoda, Nobuya Inagaki, Kenji Osafune, "iPSC technology-based regenerative therapy for diabetes," J Diabetes Investig, 9(2), 234-243, July 2018.

[15] Felicia W Pagliuca, Jeffrey R Millman, Mads Gürtler, Michael Segel, Alana Van Dervort, Jennifer Hyoje Ryu, Quinn P Peterson, Dale Greiner, Douglas A Melton, "Generation of functional human pancreatic beta cells in vitro," Cell, 159(2), 428- 439, October 2014.

[16] Jeffrey R. Millman and Felicia W. Pagliuca, "Autologous Pluripotent Stem Cell-Derived b-Like Cells for Diabetes Cellular Therapy," Diabetes, 66(5), 1111-1120. 